\title{
Human seminal fluid as a source of prostate cancer-specific microRNA biomarkers
}

\section{Dear Editor,}

Prostate cancer (PCa) is the most commonly diagnosed malignancy among men living in Western countries and a major cause of cancer-related deaths. Biopsy-based diagnosis of $\mathrm{PCa}$ is usually performed following an elevated serum prostate-specific antigen (PSA) measurement and/or abnormal digital rectal examination (DRE). The deficiencies of serum PSA as a biomarker have been well documented (Roobol \& Carlsson 2013). While it is highly specific for tissues of prostatic origin, PSA is not cancer specific, resulting in many unnecessary biopsies of benign disease. Moreover, PSA screening has resulted in substantial over-diagnosis and over-treatment of indolent tumours without having a significant effect on PCa mortality (Schroder et al. 2009). Biomarkers that could identify patients with clinically significant PCa would be ideal but are currently lacking.

Aberrant microRNA (miRNA) expression is a common feature of many diseases, including PCa (Selth et al. 2012), and there has been widespread interest in the diagnostic, prognostic and predictive potential of these molecules. Recent focus has shifted towards circulating miRNAs, which can be sourced from minimally invasive samples, such as serum, plasma and urine, and show promise as biomarkers for PCa (Sapre \& Selth 2013). Human seminal fluid (SF) is enriched for exocrine and other constituents of the prostate, such as PCa-derived cells, proteins and metabolites (Gardiner et al. 2003, Roberts et al. 2011), but is yet to be investigated as a source of PCa-associated miRNAs. In this study, we used small RNA sequencing and quantitative RT-PCR (qRT-PCR) to assess the potential of SF miRNAs as diagnostic biomarkers of PCa.

SF samples were collected from patients attending the Urology Outpatient Clinic at the Royal Brisbane and Women's Hospital following informed consent. Sample processing was conducted as described previously (Gardiner et al. 2003), and RNA was extracted using the Trizol reagent (Life Technologies) and cleaned up using RNeasy Mini kits (Qiagen), according to the manufacturer's instructions. Deep sequencing using Illumina's TruSeq Small RNA workflow was used to analyse the small RNA population in the non-sperm cellular fraction of SF, which includes prostatic epithelial, urothelial and inflammatory cells (Gardiner et al. 2003). Two RNA pools, representing groups of men with low/intermediate risk cancer (D'Amico et al. 1998) or men without cancer, were compared (Table 1). Men in both groups had elevated

Table 1 Clinical details of patient samples used in this study

\begin{tabular}{l} 
Variable \\
\hline Age, mean \pm s.E.M. (years) \\
Pre-operative PSA (ng/ml) \\
$\leq 10$ \\
$>10$ \\
Gleason score \\
6 \\
7 \\
8 \\
9
\end{tabular}

\begin{tabular}{|c|c|c|c|}
\hline \multicolumn{4}{|c|}{ Screening (RNA-seq) study } \\
\hline Total $(n=12)$ & Cancer $(n=6)$ & Control $(n=6)$ & $P$ value $^{a}$ \\
\hline $60.4 \pm 2.1$ & $58.5 \pm 3.3$ & $62.3 \pm 2.8$ & $\begin{array}{l}0.457 \\
1.000\end{array}$ \\
\hline
\end{tabular}

\begin{tabular}{|c|c|c|c|}
\hline \multicolumn{4}{|c|}{ Validation study } \\
\hline Total $(n=48)$ & Cancer $(n=26)$ & Control $(n=22)$ & $P$ value $^{\mathrm{a}}$ \\
\hline $60.9 \pm 1.3$ & $60.8 \pm 1.5$ & $61.0 \pm 2.1$ & $\begin{array}{l}0.716 \\
0.189\end{array}$ \\
\hline
\end{tabular}

\begin{tabular}{rrrrr}
10 & 5 & 5 & \\
2 & 1 & 1 & - \\
1 & 1 & - & \\
5 & 5 & & \\
- & - & & \\
- & - & & \\
\hline
\end{tabular}

$\begin{array}{r}38 \\ 11 \\ 7 \\ 14 \\ 1 \\ 4 \\ \hline\end{array}$

19
8
7
14
1
4

${ }^{\text {a } M a n n-W h i t n e y ~} U$ test or Fisher's exact test, $P$ value. 
A

\begin{tabular}{llllll}
\hline & \multicolumn{2}{c}{ Control } & & \multicolumn{2}{c}{ Cancer } \\
\cline { 2 - 3 } \cline { 5 - 6 } & Reads & $\%$ small RNA & Reads & $\%$ small RNA \\
\hline miRNA & $8,684,210$ & 37.6 & $5,003,959$ & 22.9 \\
scRNA & $1,527,018$ & 6.6 & & 971,472 & 4.5 \\
snoRNA & 762,471 & 3.3 & & 141,359 & 0.6 \\
snRNA & 200,218 & 0.9 & & 63,217 & 0.3 \\
tRNA & $11,919,255$ & 51.6 & & $15,687,212$ & 71.7 \\
Total & $23,093,172$ & 100.0 & & $21,867,219$ & 100.0
\end{tabular}
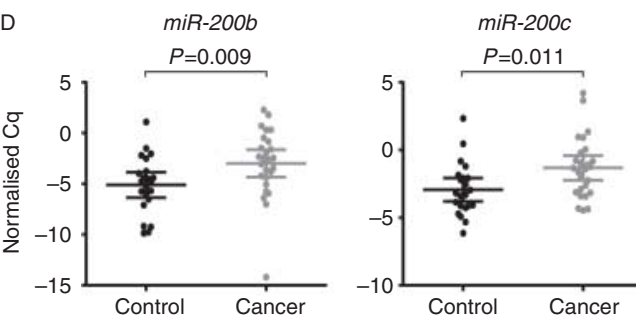

E
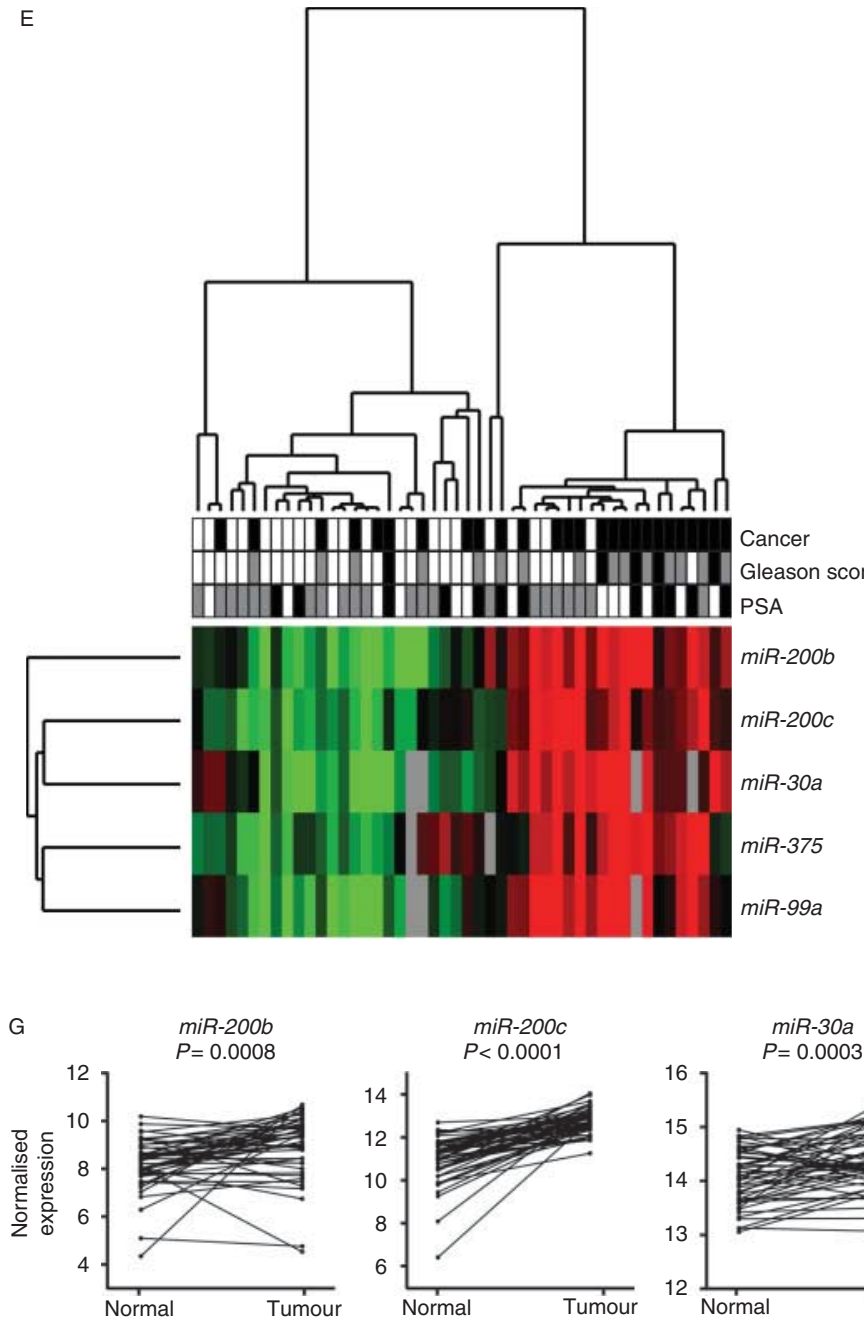
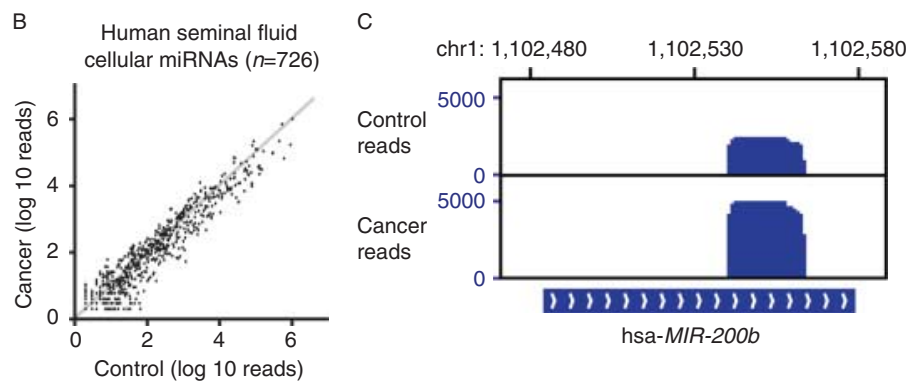

hsa-MIR-200b
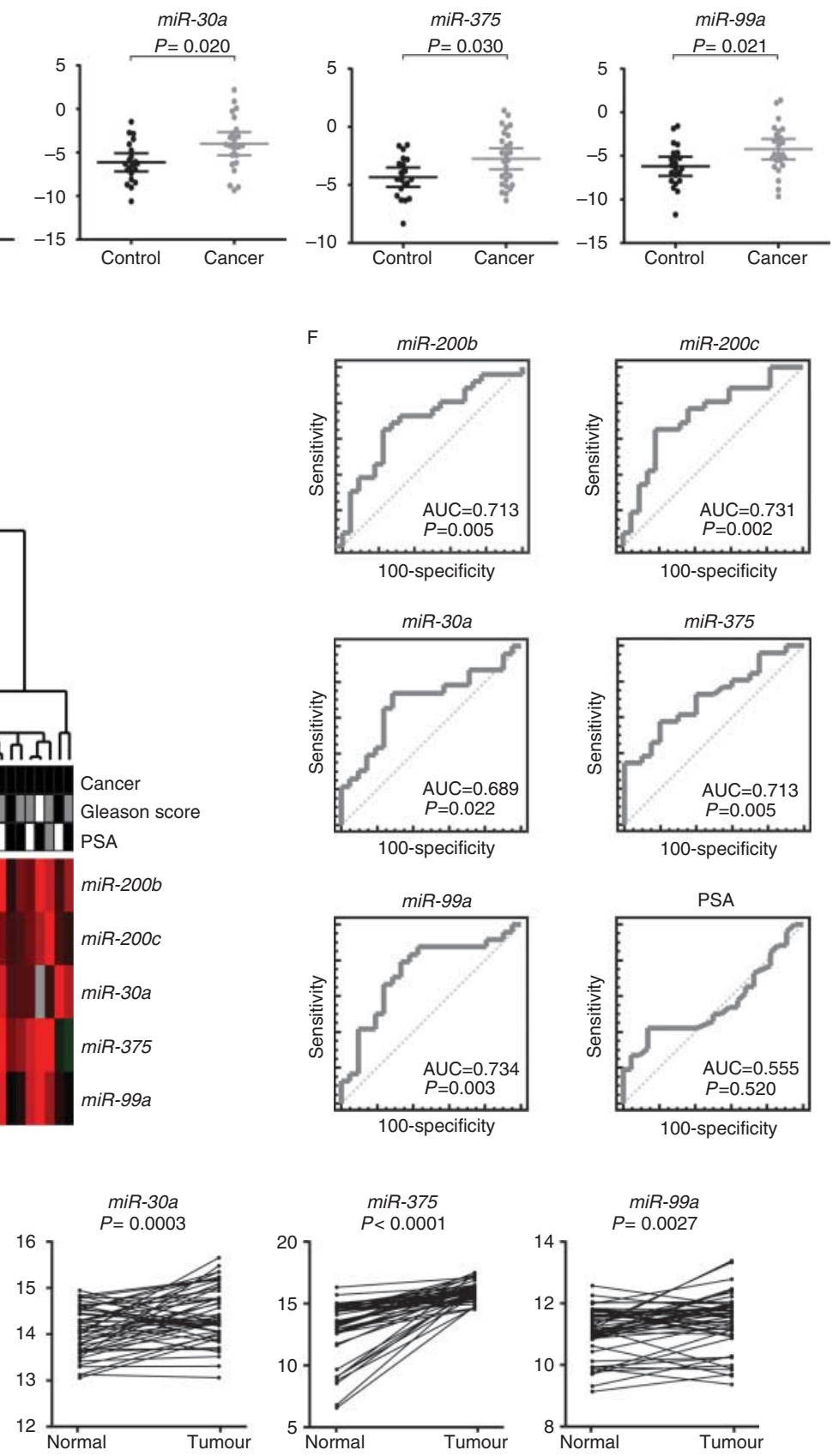

http://erc.endocrinology-journals.org 
serum PSA levels to increase the likelihood of identifying miRNA biomarkers that would provide additional diagnostic information.

RNA-seq demonstrated that the small RNA population of the non-sperm SF cellular fraction consisted primarily of tRNA and miRNA (Fig. 1A). Interestingly, a higher ratio of tRNA to miRNA was observed in cancer compared with the control. Of the 1523 miRNAs that were assessed using our workflow, 824 and 851 were detected (at least one read) in the cancer and control patients respectively. Despite the control sample exhibiting increased miRNA reads, the miRNA populations in cancer and non-cancer samples were highly concordant: 726 miRNAs were detected in both groups and the common miRNAs were generally expressed at similar levels (Fig. 1B).

To identify miRNAs that were differentially expressed in the SF of cancer patients, we considered only robustly expressed miRNAs ( $\geq 1000$ raw reads in either pool) and applied a twofold change cutoff after generating normalised expression values. This filtering strategy yielded 82 miRNAs (20 increased and 62 decreased) that were altered in PCa (data not shown). Visual inspection of RNAseq read density in individual miRNA genes confirmed the predicted changes in expression levels (Fig. 1C, and data not shown). A number of miRNAs, including miR-200 family members and miR-375, have been identified as candidate PCa biomarkers in previous studies of PCa tissues and body fluids (Selth et al. 2012), suggesting that the data were robust and biologically relevant.

Putative SF miRNA biomarkers of PCa were subsequently assessed in a validation cohort comprising 26 men with biopsy-proven, low-high-risk tumours and 22 men with elevated PSA levels but no detectable cancer on biopsy. Demographic and clinical characteristics of the validation cohort are shown in Table 1 ; there were no significant differences in age or PSA between the cancer patients and control subjects. For the validation study, we measured a subset of the potential biomarkers - namely, miR-125b, miR-141, miR-153, miR-200b, miR-200c, miR203, miR-215, miR-30a, miR-34c, miR-363-3p, miR-375, $m i R-449 a, m i R-96$ and $m i R-99 a$ - by qRT-PCR. We focused on miRNAs that were elevated in cancer for two reasons: i) known PCa-associated miRNAs were identified in this group and ii) to counter the possibility that miRNAs detected at lower concentrations in cancer were an artefact of the lower total miRNA read count in this group. The geometric mean of three small RNAs - miR-16, U6 and RNU48 (SNORD48) - that were stably expressed in the clinical samples (data not shown) was used for normalisation purposes. miRNAs with a detection rate $<80 \%$ (miR-141, miR-153, miR-215, miR-34c, miR-363-3p, miR$449 a$ and $m i R-96)$ were excluded from further analysis. Of the remaining seven miRNAs, miR-200b, miR-200c, $m i R-30 a, m i R-375$ and $m i R-99 a$ were present at significantly higher levels in the cancer samples (Fig. 1D). Hierarchical clustering based on these miRNAs yielded two major branches (Fig. 1E), one of which was characterised by higher levels of the SF miRNAs and was largely composed of men with biopsy-proven disease and the other characterised by lower SF miRNA levels and men with negative biopsies. These findings support the concept that individual SF miRNAs or an SF miRNA signature could be useful diagnostic tools.

To further evaluate the diagnostic potential of SF-derived miR-200b, miR-200c, miR-30a, miR-375 and $m i R-99 a$, we performed receiver-operating characteristic (ROC) analysis. All of the miRNAs were able to more accurately discriminate between cancer and non-cancer samples than serum PSA (Fig. 1F). However, miR-200b was the only significantly discriminate marker in a model generated by forward stepwise logistic regression $(P=0.015)$. The lack of an additive diagnostic benefit when considering multiple miRNAs could be explained by strong positive correlations in their levels; by contrast, none of the miRNAs were correlated with serum PSA (data not shown). A combination of miR-200b and serum PSA (area under the curve $(\mathrm{AUC})=0.751$ ) was significantly $(P=0.030)$ better at identifying men with cancer than PSA alone (AUC $=0.555)$, suggesting that SF miR-200b could provide complementary diagnostic information to

\section{Figure 1}

Seminal fluid miRNAs are markers of prostate cancer. (A) Small RNA-seq reads mapping to known miRNAs, tRNAs, snRNAs, snoRNAs and scRNAs in human SF. (B) Relative expression of known miRNAs with one or more reads in the non-cancer or cancer samples. (C) Screenshot illustrating alignment of reads (in blue) to the mature miRNA sequence within the MIR200b gene. The $y$-axis shows the number of mapped reads and demonstrates increased expression of miR-200b in cancer. (D) Expression of putative miRNA markers of prostate cancer in the validation cohort. Statistically significant differences were assessed using a $t$-test; $P$ values are shown. (E) Hierarchical clustering of the validation cohort based on the levels of $m i R-200 b$,
miR-200c, miR-30a, miR-375 and miR-99a in SF. Levels of miRNAs are shown in a heat map. Cancer: black, positive; white, negative. Gleason score: black, $\geq 8$; grey, 7; white, 6 and non-cancer. PSA: black, > 10; grey, 4-10; white, $<4$. (F) ROC curve analysis of miRNAs in human seminal fluid in the validation cohort. Values for AUC and statistical significance are shown. (G) Seminal fluid miRNA markers of prostate cancer are elevated in primary prostate tumours. The graphs show relative expression of the miRNAs in primary tumours and patient-matched normal prostate tissues from The Cancer Genome Atlas. Statistically significant differences were assessed using a paired $t$-test; $P$ values are shown. http://erc.endocrinology-journals.org DOI: 10.1530/ERC-14-0234
(C) 2014 Society for Endocrinology Printed in Great Britain
Published by Bioscientifica Ltd. 
current clinical tests. Moreover, SF miR-200b could distinguish between a group of men without cancer or with Gleason score (GS) 6 cancer and another group of men with $\mathrm{GS} \geq 7$ cancer (AUC $=0.666, P=0.030$ ), indicating that this marker may have prognostic potential.

To further assess the biological relevance of the putative SF miRNA biomarkers, we interrogated a publically available dataset from The Cancer Genome Atlas, which contains miRNA expression data for prostate tumours and patient-matched benign tissues ( $n=50$ of each). The five putative SF miRNA biomarkers were expressed in both malignant and benign samples, and all were significantly elevated in cancer (Fig. 1G). This analysis suggests that the miRNAs identified in this study are likely to be derived from prostatic tumour cells within the SF.

Biomarkers that can accurately detect PCa at an early stage and identify aggressive disease subtypes are urgently required to improve patient management. In this study, we provide proof-of-principle that SF cellular material from patients is a potentially useful source of miRNA diagnostic biomarkers. Specifically, SF-derived miR-200b, $m i R-200 c, m i R-30 a, m i R-375$ and $m i R-99 a$ were found at higher levels in men with elevated PSA levels and biopsyproven cancer compared with men with elevated PSA levels but no cancer. Moreover, SF miR-200b was also associated with Gleason score and therefore may have prognostic value. Further investigation of these potential biomarkers in a larger cohort is now warranted.

Robust and validated SF-derived miRNA diagnostic biomarkers could potentially be useful in a number of clinical scenarios. First, the complementary information provided by such markers may help to guide the urgency of biopsy for men with elevated serum PSA levels or a concerning DRE result. For example, men with low levels of SF PCa miRNA biomarkers such as miR-200b may benefit from postponing biopsy and vice versa. In such a situation, SF miRNAs could be used in combination with other emerging molecular tools, such as urine TMPRSS2:ERG and PCA3 (Tomlins et al. 2011), to provide a more robust appraisal of the likelihood of clinically significant disease. Secondly, SF miRNAs could have a role as a non-invasive tool for monitoring men in active surveillance regimens, a management approach now adopted worldwide for low-risk PCa (Dall'Era et al. 2012). While the advantages of active surveillance are clear, it can be compromised by serum PSA testing, biopsy undersampling and molecular heterogeneity of histologically inapparent multifocal disease (Haas et al. 2007, Dall'Era et al. 2012). SF miRNA measurements could be used to help determine if or when to proceed with curative interventions by providing an additional measure of global prostatic pathology, with a clear advantage of repeated and non-invasive sampling.

A limitation of this study is the size of the validation cohort, which did not permit robust associations between SF miRNAs and clinical parameters within the cancer group. We are in the process of accruing a larger, more representative cohort of samples to validate the findings from this study. Nevertheless, we believe that our findings provide a solid platform for the future investigation of SF-derived miRNAs as diagnostic biomarkers of PCa.

$$
\begin{array}{r}
\text { Luke A Selth }{ }^{1,2} \\
\text { Matthew J Roberts }^{3,4} \\
\text { Clement W K Chow }^{3,5} \\
\text { Villis R Marshall }^{1,2} \\
\text { Suhail A R Doi } \\
\text { Andrew D Vincent }^{6,7} \\
\text { Lisa M Butler } \\
\text { Martin F Lavin } \\
\text { 3,5 } \\
\text { Wayne D Tilley }^{1,2} \\
\text { Robert A Gardiner }^{3,4}
\end{array}
$$

${ }^{1}$ Dame Roma Mitchell Cancer Research Laboratories and Adelaide Prostate Cancer Research Centre, School of Medicine, The University of Adelaide, Level 4 Hanson Institute Building, DX Number 650 801Adelaide, South Australia, SA 5005, Australia

${ }^{2}$ Freemasons Foundation Centre for Men's Health, The University of Adelaide, Adelaide, South Australia, Australia ${ }^{3}$ Royal Brisbane Hospital, University of Queensland Centre for Clinical Research, Level 6, Building 71/918, Herston, Brisbane, Queensland, QLD 4006, Australia

${ }^{4}$ Department of Urology, Royal Brisbane and Women's Hospital, Brisbane, Queensland, Australia ${ }^{5}$ Queensland Institute of Medical Research, Herston, Brisbane, Queensland, Australia

${ }^{6}$ School of Population Health, The University of Queensland, Brisbane, Queensland, Australia

${ }^{7}$ Department of Endocrinology, Princess Alexandra Hospital, Brisbane, Queensland, Australia

(Correspondence should be addressed to L A Selth; email: luke.selth@adelaide.edu.au W D Tilley; email: wayne.tilley@adelaide.edu.au R A Gardiner; email: f.gardiner@uq.edu.au) 


\section{Declaration of interest}

The authors declare that there is no conflict of interest that could be perceived as prejudicing the impartiality of the research reported.

\section{Funding}

This work was supported by Young Investigator Awards from the Prostate Cancer Foundation (the Foundation 14 award; L A Selth) and the Prostate Cancer Foundation of Australia (PCFA) (L A Selth, YI 0810), PCFA/Cancer Australia (Grant ID 1012337 to L M Butler and W D Tilley), the RBWH Foundation (to R A Gardiner), and PCFA/Cancer Council Queensland (to R A Gardiner). M J Roberts was supported by a Doctor in Training Research Scholarship from Avant Mutual Group Ltd. The Adelaide Prostate Cancer Research Centre was supported by an establishment grant from the PCFA (ID 2011/0452). L M Butler was supported by a senior research fellowship from the Cancer Council of South Australia.

\section{Acknowledgements}

Ethical approval for the study was obtained from the Royal Brisbane and Women's Hospital Human Research Ethics Committee (HREC/09/QRBW/320, HREC/09/QRBW/305 and 1995/088B) and the University of Queensland Medical Research Ethics Committee (Project no. 2006000262). The authors thank the study participants, and the urologists, nurses and histopathologists who assisted in the recruitment and collection of patient material and information. The results published here are in part based on the data generated by The Cancer Genome Atlas, established by the National Cancer Institute and the National Human Genome Research Institute, and they are grateful to the specimen donors and relevant research groups associated with this project.

\section{References}

Dall'Era MA, Albertsen PC, Bangma C, Carroll PR, Carter HB, Cooperberg MR, Freedland SJ, Klotz LH, Parker C \& Soloway MS 2012 Active surveillance for prostate cancer: a systematic review of the literature. European Urology 62 976-983. (doi:10.1016/j.eururo. 2012.05.072)
D'Amico AV, Whittington R, Malkowicz SB, Schultz D, Blank K, Broderick GA, Tomaszewski JE, Renshaw AA, Kaplan I, Beard CJ et al. 1998 Biochemical outcome after radical prostatectomy, external beam radiation therapy, or interstitial radiation therapy for clinically localized prostate cancer. Journal of the American Medical Association 280 969-974. (doi:10.1001/jama.280.11.969)

Gardiner RA, Burger M, Clements JA \& Lavin MF 2003 Realizing the potential of ejaculate/seminal fluid in detecting and predicting natural history. Methods in Molecular Medicine 81 199-217. (doi:10.1385/1-59259-372-0:199)

Haas GP, Delongchamps NB, Jones RF, Chandan V, Serio AM, Vickers AJ, Jumbelic M, Threatte G, Korets R, Lilja H et al. 2007 Needle biopsies on autopsy prostates: sensitivity of cancer detection based on true prevalence. Journal of the National Cancer Institute 99 1484-1489. (doi:10.1093/jnci/djm153)

Roberts MJ, Schirra HJ, Lavin MF \& Gardiner RA 2011 Metabolomics: a novel approach to early and noninvasive prostate cancer detection. Korean Journal of Urology 52 79-89. (doi:10.4111/kju.2011. 52.2.79)

Roobol MJ \& Carlsson SV 2013 Risk stratification in prostate cancer screening. Nature Reviews. Urology 10 38-48. (doi:10.1038/nrurol. 2012.225)

Sapre N \& Selth LA 2013 Circulating microRNAs as biomarkers of prostate cancer: the state of play. Prostate Cancer 2013 539680. (doi:10.1155/ 2013/539680)

Schroder FH, Hugosson J, Roobol MJ, Tammela TL, Ciatto S, Nelen V, Kwiatkowski M, Lujan M, Lilja H, Zappa M et al. 2009 Screening and prostate-cancer mortality in a randomized European study. New England Journal of Medicine 360 1320-1328. (doi:10.1056/ NEJMoa0810084)

Selth LA, Tilley WD \& Butler LM 2012 Circulating microRNAs: macroutility as markers of prostate cancer? Endocrine-Related Cancer 19 R99-R113. (doi:10.1530/ERC-12-0010)

Tomlins SA, Aubin SM, Siddiqui J, Lonigro RJ, Sefton-Miller L, Miick S, Williamsen S, Hodge P, Meinke J, Blase A et al. 2011 Urine TMPRSS2:ERG fusion transcript stratifies prostate cancer risk in men with elevated serum PSA. Science Translational Medicine 3 94ra72. (doi:10.1126/scitranslmed.3001970)

Received in final form 9 May 2014

Accepted 19 May 2014

Made available online as an Accepted Preprint

23 May 2014 http://erc.endocrinology-journals.org DOI: 10.1530/ERC-14-0234
(C) 2014 Society for Endocrinology Printed in Great Britain
Published by Bioscientifica Ltd 